\title{
Comparison of Retinal Thickness Measurements of Normal Eyes between Topcon Algorithm and a Graph Based Algorithm
}

\author{
Enting Gao ${ }^{1,2}$, Fei Shi ${ }^{1}$, Weifang Zhu ${ }^{1}$, Binyao Chen $^{3}$, Haoyu Chen ${ }^{3}$,Xinjian \\ Chen $^{1 *}$ \\ 1School of Electrical and Information Engineering, Soochow University, Suzhou, China \\ 2School of Electronic and Information Engineering, Suzhou University of Science and \\ Technology, Suzhou, China \\ 3Joint Shantou International Eye Center, Shantou University and the Chinese University of \\ Hong Kong, Shantou, China
}

\begin{abstract}
To assess the agreement between Topcon built-in algorithm and our developed graph based algorithm, the retinal thickness of 9-sectors on an Early Treatment of Diabetic Retinopathy Study(ETDRS) chart measurements for normal subjects was compared. A total of fifty eyes were enrolled in this study. The overall and sectoral thickness on ETDRS chart were calculated using Topcon built-in algorithm and our developed three-dimensional graph based algorithm. Correlation analysis and agreement analysis were performed between the commercial algorithm and our algorithm. A high degree of correlation was found between the results obtained from the two methods was from 0.856 to 0.960 . It's showed that our developed graph based algorithm can provide excellent performance similar to Topcon algorithm.
\end{abstract}

Keywords: Algorithms Comparison, Graph-Search, Retinal Layer Segmentation.

\section{Introduction}

For clinicians managing patients with different retinal diseases, retinal thickness is often measured by optical coherence tomography (OCT) and followed as a means of objectively monitoring the development of retinal diseases. For example, retinal nerve fiber of glaucoma patients are gradually damaged and lost, leading to thinning of the retinal nerve fiber layer (RNFL)[1, 2]. The structural parameter of the optic nerve head(ONH) and retinal RNFL thickness measured from OCT is often used for the evaluation of glaucoma[3]. Therefore it is very important for the ophthalmologist to have a reliable and efficient method to quantitatively analyze the retinal structural parameter.

Optical coherence tomography (OCT) is the most commonly used imaging technology for macula examination. Especially the recent developed spectral domain

* Corresponding author. Address: No.1, Shizi Street, Suzhou, Jiangsu, P.R. China 215006. Tel.: +86-18260180695. Email: xjchen@suda.edu.cn 
OCT (SD-OCT) can provide non-invasive, in vivo, high speed and high resolution three-dimensional imaging of anterior and posterior eye structure.[4]

Most commercially available SD-OCT can provide only the peripapillary RNFL thickness and macula-centered retinal thickness. For example, Topcon 3D-OCT 2000, can only calculate the thickness for the following retinal layers: RNFL, GCL+IPL, IS/OS and RPE. Therefore its discriminating ability is limited. We have developed an accurate and reliable measurement of the thickness for all 10 retinal layers on maculacentered SD-OCT.

Although there are a few published studies that have compared the retinal layer thickness measurements between different softwares, most of them are comparisons between commercial softwares[5-8]. In this study, we compared the commercially available Topcon in-built algorithm and our developed graph based algorithm by comparing the retinal thickness of 9-sectors on ETDRS measurements for normal subjects. It can provide a reference standard for analyzing patients with different retinal diseases.

\section{$2 \quad 3 D-O C T$ Image analysis}

The proposed method consists of three phases: retinal layer segmentation, 9-sectors dividing and statistical analysis. Figure 1 shows the flowchart of the proposed method. The retinal layer segmentation consists of two main steps: preprocessing and layer segmentation.During the 9-sectors dividing phase, macula-centered retinal image is divided into 9 sectors by use of the Early Treatment Diabetic Retinopathy (ETDRS) chart[9]. Finally, the thickness of each sector is calculated and analyzed.

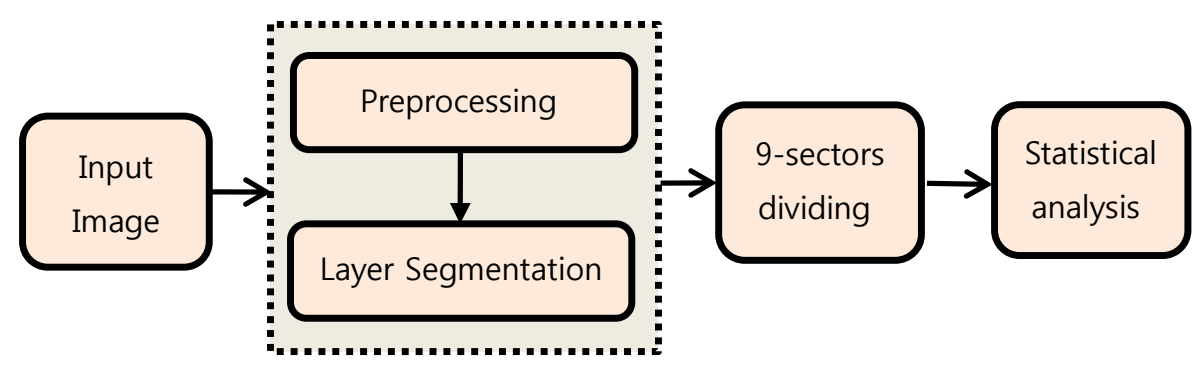

Figure 1. The flowchart of the proposed 3D OCT Imaging analysis system.

\subsection{Preprocessing}

During the preprocessing part, first a curvature anisotropic diffusion filter was used to reduce the speckle noise in OCT images. Then, five multiscale OCT volumes are created by subsampling by a factor of 2 in the z-axis. Finally, the gradient magnitude in $\mathrm{z}$ direction was calculated as a cost function. 


\subsection{Retinal Layer Segmentation Using Multiscale 3D Graph Search}

The graph search approach employed here transforms the intraretinal layers segmentation problem into an optimal surface search problem[10, 11], which was then transformed to finding a minimum cost closed set in a vertex-weighted directed graph. A weighted directed graph $\mathrm{G}=(\mathrm{v}, \mathrm{e})$ is constructed which composes of a node set $\mathrm{V}$ and an arc set $\mathrm{E}$. In the graph, the nodes $\mathrm{v} \in \mathrm{V}$ correspond to image voxels and $\operatorname{arcs}\left\langle\mathrm{v}_{\mathrm{i}}, \mathrm{v}_{\mathrm{j}}\right\rangle \in \mathrm{E}$ connect the nodes vi,vj. The cost(or weight) of the node $\mathrm{v} \in \mathrm{V}$ is derived from the cost function that can be expressed as some measure such as gradients that the corresponding voxels belong to the surface[12]. By finding an optimal closed set in a vertex-weighted graph, the approach was able to segment the intraretinal surfaces. In the higher resolution, the surfaces are detected near the locations obtained from the next lower resolution.

The commercially available Topcon in-built algorithm (Topcon 3D-OCT 2000, 8.11.003.04) is a two-dimension approach, and only 4 intraretinal surfaces (ILM, NFL, IPL, RPE) can be obtained in 3D Macular Mode in Macular B-scan (Figure 2(a)). Eleven surfaces of the 3D-OCT volumetric macula-centered scan were segmented using the multi-resolution graph search algorithm (Figure 2(b)).

For Topcon, the Thickness of the 9-Sectors on ETDRS Chart of macular-central retinal were obtained from the Topcon in-built algorithm.

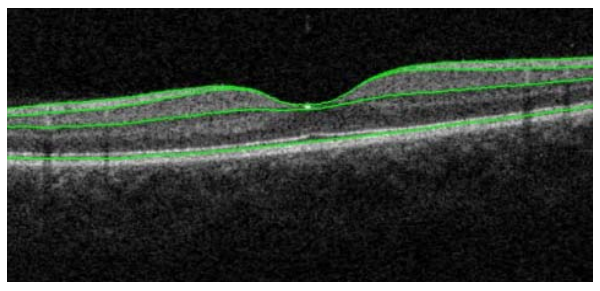

a

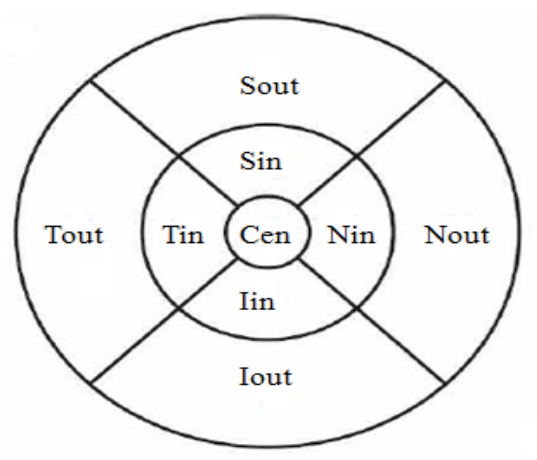

C

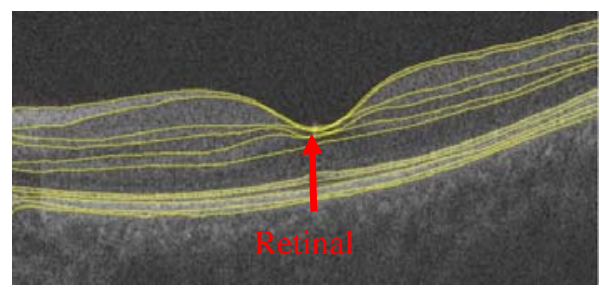

b

Figure 2. Computation of macular thickness in 9- sectors on ETDRS chart. (a) Macular B-scan with intraretinal surfaces ILM, NFL, IPL, RPE identified in 3D Macular Mode by Topcon algorithm. (b) Macular B-scan with intraretinal layers ILM, NFL, GCL, IPL, INL, OPL, ONL, IS/OS, RPE identified by our graph based algorithm. (c) ETDRS scheme applied for retinal thickness measurement. The area of Cen represents the fovea. Mean retinal thickness was calculated for superior(S), inferior(I), temporal(T), nasal(N) with the diameter of $1 \mathrm{~mm}$, $3 \mathrm{~mm}, 6 \mathrm{~mm}$. 


\section{3 macular-central retinal thickness of the 9-Sectors on ETDRS Chart}

In this step, we first found the lowest location of the first surface (ILM) which is used as a center point of Early Treatment Diabetic Retinopathy (ETDRS) chart[9]. The ETDRS plot includes three circles which divided the macula into two rings. The inner circle of the ETDRS plot has a diameter of $1 \mathrm{~mm}$, the middle circle of the ETDRS plot has a diameter of $3 \mathrm{~mm}$, the outer circle of the ETDRS plot has a diameter of $6 \mathrm{~mm}$, and these circle were fovea centered. Then the macula was further divided into four quadrants: Tout and Tin correspond to outer and inner of temporal quadrants, Nout and Nin correspond to outer and inner of nasal quadrants, Sout and Sin correspond to outer and inner of superior quadrants, and areas Iout and Iin correspond to outer and inner of inferior quadrants. (Figure1 (c)). Finally, the information of surface1 and furface11 were utilized, The thickness of all 9-sectors on ETDRS chart of macula-centered retina were measured.

\section{$2.4 \quad$ Statistical analysis}

Mean thickness of macular-central retinal of 9-sectors on ETDRS chart from two different algorithms were analyzed with Pearson's correlation. Correlation coefficient(r) and mean differences were calculated. Bland-Altman analysis was performed in order to assess the agreement between the Topcon and our measurements. The software SPSS (Version 16.0, IBM), MedCalc (http://www.medcalc.org/) was used to conducted statistical analysis.

\section{Experimental Results}

\subsection{Subjects}

A total of 50 normal subjects were enrolled in the study, and one eye was randomly selected for each subject. All subjects were examined at the Institutional Review Board of Joint Shantou International Eye Center (JSIEC), Shantou University and the Chinese University of Hong Kong from August 2013 to March 2014. There were 27 males/23 females normal subjects age ranged from 20 to 60 years old. All eligible subjects received explanation of the study and signed an informed consent form in accordance with the principles embodied in the Declaration of Helsinki.

Detailed medical histories were taken from all subjects. They underwent complete ophthalmic examinations including best corrected acuity, intraocular pressure, refractive error, slit-lamp biomicroscopy, fundus examination, visual field (VF) and optical coherence tomography (OCT) scan. Inclusion criteria consisted of a bestcorrected visual acuity (BCVA) of 0.5 or better, intraocular pressure $\leqslant 21 \mathrm{mmHg}$, spherical refraction between -6.0 to 6.0 diopters (D), normal optic disc appearance and absence of other ocular diseases. One eye was randomly chosen if both eyes were eligible. 
Table 1. Characteristics of Included Subjects

\begin{tabular}{ll}
\hline & Normal \\
\hline Sex(male/female) & $27 / 23$ \\
Age(years) & $32.06 \pm 10.24$ \\
SE refraction(D) & $-0.61 \pm 1.47$ \\
Axial Length(mm) & $22.17 \pm 1.42$ \\
IOP(mmHg) & $15.32 \pm 2.61$ \\
MD(dB) & $-1.1 \pm 1.21$ \\
PSD(dB) & $1.45 \pm 0.49$ \\
\hline
\end{tabular}

Table 2. Comparison of Retinal Thickness of 9-sectors on ETDRS Measured by Topcon Algorithm and graph based Algorithm

\begin{tabular}{cccccc}
\hline & $\begin{array}{c}\text { Topcon } \\
\text { algorithm }\end{array}$ & $\begin{array}{c}\text { Graph based } \\
\text { algorithm }\end{array}$ & $\begin{array}{c}\text { Correlation } \\
\text { coefficient }\end{array}$ & p-value & $\begin{array}{c}\text { Mean Difference } \\
\text { (Topcon-Graph based) }\end{array}$ \\
\hline Cen & $224.42 \pm 16.03$ & $217.31 \pm 15.02$ & 0.960 & $<0.0001$ & 7.11 \\
Sin & $305.24 \pm 14.49$ & $301.61 \pm 14.42$ & 0.954 & $<0.0001$ & 3.63 \\
Nin & $306.56 \pm 14.86$ & $301.11 \pm 13.67$ & 0.892 & $<0.0001$ & 5.45 \\
Iin & $299.88 \pm 13.97$ & $297.23 \pm 12.80$ & 0.899 & $<0.0001$ & 2.65 \\
Tin & $287.84 \pm 14.43$ & $287.56 \pm 14.19$ & 0.950 & $<0.0001$ & 0.28 \\
Sout & $270.84 \pm 13.87$ & $266.97 \pm 14.03$ & 0.895 & $<0.0001$ & 3.87 \\
Nout & $287.22 \pm 15.12$ & $290.21 \pm 18.89$ & 0.856 & $<0.0001$ & 2.99 \\
Iout & $257.62 \pm 12.11$ & $254.45 \pm 13.60$ & 0.885 & $<0.0001$ & 3.17 \\
Tout & $253.50 \pm 13.44$ & $248.12 \pm 16.44$ & 0.865 & $<0.0001$ & 5.38 \\
Ave & $273.34 \pm 11.76$ & $265.18 \pm 12.05$ & 0.943 & $<0.0001$ & 8.16 \\
\hline
\end{tabular}

The raw data were obtained from Topcon 3D-OCT 2000, macula-centered and optic nerve head $(\mathrm{ONH})$-centered SD-OCT volumes were acquired. Each SD-OCT volume was $512 \times 128 \times 885$ voxels, corresponding to physical dimensions of $6 * 6 * 2.3 \mathrm{~mm}^{3}$, SD-OCT volumes with image quality lower than 45 were excluded.

\subsection{Rusults}

The average age and image quality score of 50 normal subjects were $32.06 \pm 10.24$ and 57.46 \pm 3.99 , respectively. population characteristics were summarized in Table 1.

Mean thickness (microns) of 9-sectors on ETDRS reported are (Topcon Algorithm vs graph based Algorithm):Cen (224.42 vs 217.31, $\mathrm{P}<0.0001)$; $\operatorname{Sin}(305.24$ vs 301.61 , $\mathrm{P}<0.0001)$; Nin (306.56 vs 301.11, $\mathrm{P}<0.0001)$; $\operatorname{Iin}(299.88$ vs 297.23, $\mathrm{P}<0.0001$ ) ; Tin(287.84 vs 287.56, $\mathrm{P}<0.0001)$; Sout(270.84 vs, $266.97 \mathrm{P}<0.0001)$; $\operatorname{Nout(287.22}$ vs 20.21, $\mathrm{P}<0.0001)$; Iout(254.45 vs 251.64, $\mathrm{P}<0.0001)$; $\operatorname{Tout}(253.50$ vs 248.12 , $\mathrm{P}<0.0001)$; Ave(273.34 vs 265.18, $\mathrm{P}<0.0001)$ (Table2) .

A high degree of correlation was found between the results obtained from the two methods as evidenced in Table2 and Figure 3, with $r>0.856$ and all $p<0.0001$. However, the mean differences of Cen and Ave were 7.11 and 8.16 - slightly 
higher but not significantly different as demonstrated by the overlapping 95\% confidence intervals (Table 2 and Figure 3)
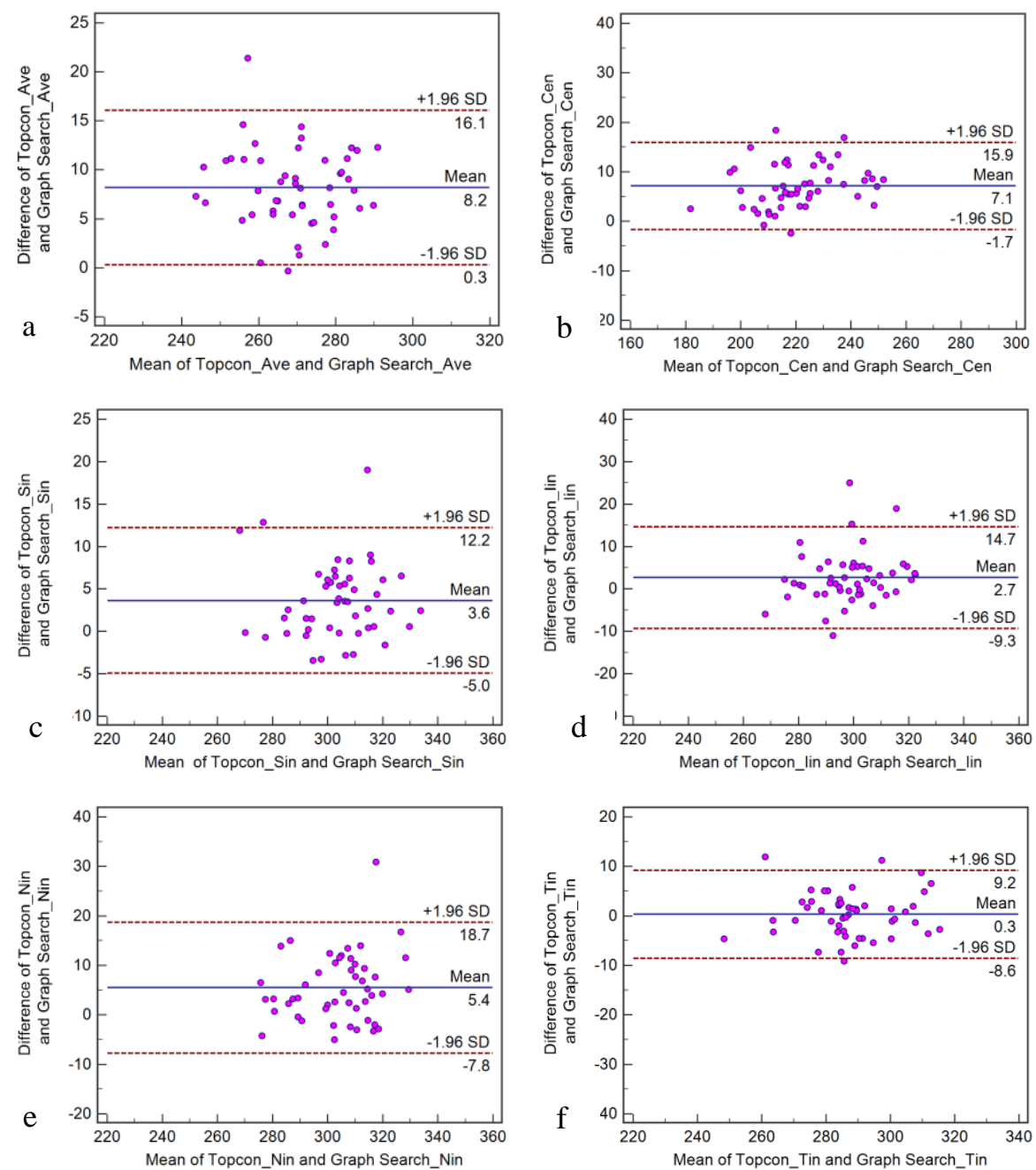

Figure 3. Bland-Altman plot. (a) total macular thickness (Ave), (b) fovea thickness (Cen), (c) superior inner thickness (Sin), (d) inferior inner thickness (Iin), (e) nasal inner thickness (Nin) and (f) temporal inner thickness (Tin) difference between Topcon algorithm and graph based algorithm against the average of the two.

\section{Conclusions and discussions}

The presented study compared the retinal thickness of 9-sectors on ETDRS chart that measured by the latest Topcon 3D-OCT 2000 built-in algorithm and our graph 
based algorithm for normal subjects. The results from this study showed that our graph based algorithm has good performance similar to Topcon built-in algorithm.

A high degree of correlation was found between Topcon built-in algorithm and graph search algorithm, which were used to determine the retinal thickness of 9sectors on ETDRS in the normal eyes. The correlation of two methods in Nout and Tout is slightly lower than other subfields, which may be related to the measurement error caused by slant retinal images. Bland-Altman analysis revealed that there was good agreement between Topcon built-in algorithm and our graph based algorithm for the thickness measurements. However, some plots in Figure 3 showed greater difference. The reason may be that the Topcon built-in algorithm resulted in significant segmentation error for some data while our graph based algorithm segmented them successfully (Figure 3, Figure 4).

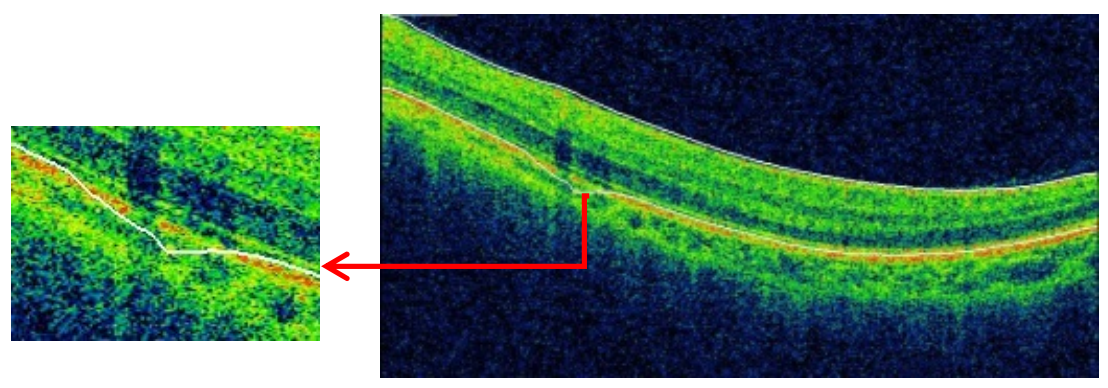

Figure 4. Segmentation error using Topcon algorithm in normal subjects.

In summary, our graph based algorithm has provided an excellent segmentation and measurement performance in normal subjects comparable to that of the Topcon algorithm. Moreover, our graph based algorithm can segment retinal into more layers than the Topcon buit-in algorithm, so it can provide more layer thickness information for the diagnosis and treatment of eye diseases.

\section{References}

[1] H.A. Quigley, E.M. Addicks, W.R. Green, "Optic nerve damage in human glaucoma: III. Quantitative correlation of nerve fiber loss and visual field defect in glaucoma, ischemic neuropathy, papilledema, and toxic neuropathy", Archives of Ophthalmology, 100,pp135146,1982.

[2] F.N. Kanadani, D.C. Hood, T.M. Grippo, B. Wangsupadilok, N. Harizman, V.C. Greenstein, J.M. Liebmann, R. Ritch, Structural and functional assessment of the macular region in patients with glaucoma, British journal of ophthalmology, 90,pp1393-1397,2006.

[3] C.K. Leung, W.-M. Chan, W.-H. Yung, A.C. Ng, J. Woo, M.-K. Tsang, R.K. Tse, Comparison of macular and peripapillary measurements for the detection of glaucoma: an optical coherence tomography study, Ophthalmology, 112,pp391-400,2005.

[4] G.L. Trick, F.Y. Calotti, B. Skarf, Advances in imaging of the optic disc and retinal nerve fiber layer, Journal of neuro-ophthalmology, 26, pp284-295,2006.

[5] U.E. Wolf-Schnurrbusch, L. Ceklic, C.K. Brinkmann, M.E. Iliev, M. Frey, S.P.

Rothenbuehler, V. Enzmann, S. Wolf, Macular thickness measurements in healthy eyes using six different optical coherence tomography instruments, Investigative ophthalmology \& visual 
science, 50,pp3432-3437,2009.

[6] C.K.-s. Leung, C.Y.-l. Cheung, R.N. Weinreb, G. Lee, D. Lin, C.P. Pang, D.S. Lam, Comparison of macular thickness measurements between time domain and spectral domain optical coherence tomography, Investigative ophthalmology \& visual science, 49,pp48934897,2008.

[7] O. Knight, R.T. Chang, W.J. Feuer, D.L. Budenz, Comparison of retinal nerve fiber layer measurements using time domain and spectral domain optical coherent tomography, Ophthalmology, 116, pp1271-1277,2009.

[8] C.K.-S. Leung, V. Chiu, R.N. Weinreb, S. Liu, C. Ye, M. Yu, C.Y.-l. Cheung, G. Lai, D.S.-C. Lam, Evaluation of retinal nerve fiber layer progression in glaucoma: a comparison between spectral-domain and time-domain optical coherence tomography, Ophthalmology, 118,pp1558$1562,2011$.

[9] D.R.S.R. Group, Photocoagulation for diabetic macular edema. Early Treatment Diabetic Retinopathy Study report number 1, Arch ophthalmol, 103,pp1796-1806,1985.

[10] X. Chen, M. Niemeijer, L. Zhang, K. Lee, M.D. Abràmoff, M. Sonka, Three-dimensional segmentation of fluid-associated abnormalities in retinal OCT: probability constrained graphsearch-graph-cut, Medical Imaging, IEEE Transactions on, 31,pp1521-1531,2012.

[11] M.K. Garvin, M.D. Abràmoff, X. Wu, S.R. Russell, T.L. Burns, M. Sonka, Automated 3-D intraretinal layer segmentation of macular spectral-domain optical coherence tomography images, Medical Imaging, IEEE Transactions on, 28,pp1436-1447,2009.

[12] K.M. Lee, Segmentations of the intraretinal surfaces, optic disc and retinal blood vessels in 3D-OCT scans, Theses and Dissertations, 247,2009. 